\title{
JomNetwork: GLBP in Medium Size Enterprise
}

\author{
Siew, J. X.1 Josephng, P. S.1 , Lim, J. T.2 \\ 1School of Computing \& Creative Media, KDU University College, \\ 1Jalan Kontraktor, Seksyen, Shah Alam, Selangor, Malaysia \\ ${ }^{2}$ Faculty of Information \& Communication Technology, \\ 2University Tunku Abdul Rahman, Kampar, Perak, Malaysia
}

\begin{abstract}
Nowadays network play a very important role, every company need network. It is because, there are a lot of work done by network based and need to monitor through network. Once network of a company down it will affect the whole company. Either lower down the productivity or the whole company have to stop working. Normally in a big company they can get the most expensive and good solution for their company because Big company have sufficient money to get the best solution but for medium size company they do not have much money. Therefore, in this paper will provide a best high availability solution for medium size enterprise.
\end{abstract}

Keywords: VVRP; HSRP; GLBP; ICT infrastructure; high availability; business continuity; network; routing protocol; Small and Medium Sized Enterprise

\section{INTRODUCTION ON THE IMPACT OF MEDIUM SIZED ENTERPRISES (MSEs)}

First Hop Redundancy Protocols also known as FHRPs is a redundancy protocol. What this protocol is used for is to help shield the default gateway against a sole point of failure and to also help with doing load balancing if there are more than a single uplinks that are available at the first- hop routers. Some examples of the First Hop Redundancy Protocols include HSRP (Hot Standby Router Protocol), VRRP (Virtual Router Redundancy Protocol), and GLBP (Gateway Load Balancing Protocol). "Although MSEs being relative smaller in both user based and ICT infrastructure, this does not mean low technology adoptions" [1]. The research aims to deliver the following objectives as shown in

Table 1.

Table 1 : Research Objective

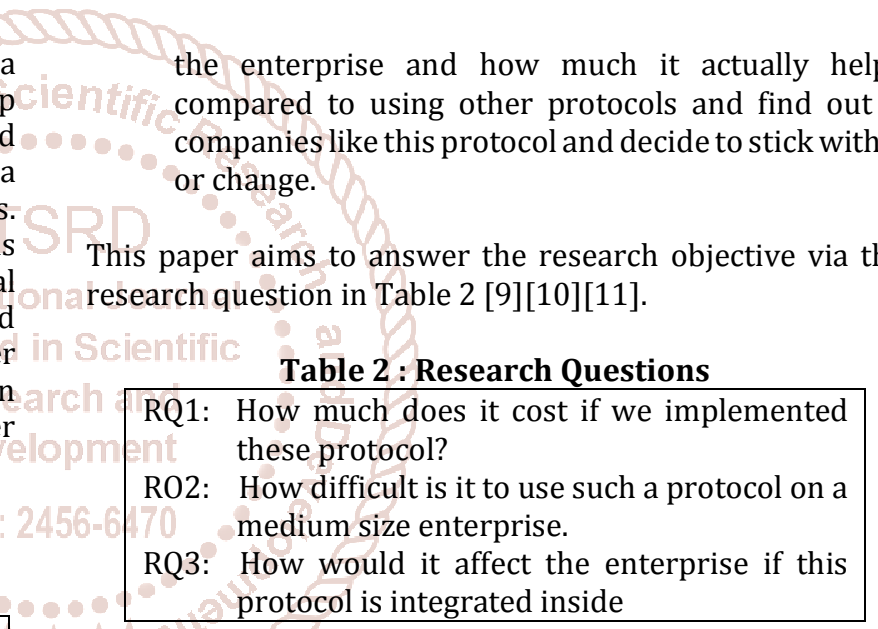

R01: To find out the cost it would take to implement the protocol

R02: To find out how much work or hassle it would be to implement such protocol

R03: To discover the satisfaction enterprise would gain from this protocol

RQ1: Compare with VRRP and HSRP this protocol might more difficult to configure than others protocol but even if it is difficult to configure, the company doesn't need to hire a network expert to maintain it, a fresh graduate network major student is able to maintain and handle it. So the cost would only be the pay of the new staff or fresh graduate and also the price of the router capable of GBLP which cost around 175 USD. actually implement GLBP in a medium sized enterprise. This is to make sure that it would not cost too much for a medium sized enterprise and that it is actually feasible to implement such a protocol at that level.

R02: To see if it is too hard to handle for a medium sized enterprise. To check if the workload would be too harsh or hard to implement for them.

R03: To find out if medium sized enterprise is pleased with the implementation of GLBP and its performance for the enterprise. And to find out what role GLBP plays in

RQ2: For a medium sized company, it would actually not be too hard to implement this protocol. However, it also depends on the enterprise's amount of network expert they have. If the enterprise only has 1 network expert that handles it, it might be too tough for him.

RQ3: GLBP would result in able to achieve load balancing. This would make it efficient in using its resources and also increase its availability to high. GLBP would make enterprise have less trouble and chance to face failure or network being not available. 
Table 3 : First Hop Redundant Protocol Matrix

\begin{tabular}{|c|c|c|}
\hline & Advantages & Disadvantages \\
\hline VRRP & $\begin{array}{l}\text {-Simple network management } \\
\text {-High adaptability } \\
\text {-Low network overhead } \\
\text {-Free patent protocol }\end{array}$ & -Not secured \\
\hline HSRP & $\begin{array}{l}\text {-Easy to configure } \\
\text {-Traffic is minimal }\end{array}$ & $\begin{array}{l}\text {-Recovery time speed } \\
\text {-Weak security } \\
\text {-Cisco proprietary protocol }\end{array}$ \\
\hline GLBP & $\begin{array}{l}\text {-Resources used efficiently } \\
\text {-High availability } \\
\text {-Automatic load balancing } \\
\text {-Low administration cost } \\
\text {-Simple access layer design }\end{array}$ & $\begin{array}{l}\text {-Cisco proprietary protocol } \\
\text {-High complexity }\end{array}$ \\
\hline
\end{tabular}

\section{VRRP (Virtual Router Redundancy Protocol) VRRP Advantages}

The first advantage of VRRP is that it has a simple network management. VRRP has a simplified network management. It is easy to deploy VRRP on broad cast LANs and multicast such as Ethernet. You can make sure that the system will be able to contribute default links which are highly reliable without changing any configurations. For example protocols such as route discovery protocols or dynamic routing protocols. If a device fails, it will prevent the network interruption because of a single link failure.

VRRP also has a high adaptability making it suitable in a lot of situations. The VRRP packets will be encapsulated in an IP packet, it will then be supported by different kinds of upper layer protocols.

Besides that, VRRP has a low network overhead. VRRP only defines one packet type. VRRP advertisement can only be sent out by the master in a VRRP group.

\section{VRRP Disadvantages}

The disadvantage VRRP is that is has a low security. No security method is used, making the authentication method of VRRP weak.

\section{HSRP (Hot Standby Router Protocol) HSRP Advantages}

HSRP is fairly easy to configure, this is because the protocols does not have any effect on the host's configuration or routing tables.

Another advantage of HSRP is that the traffic is minimal. HSRP protocol increase the traffic by a small level making it not affecting the traffic flow and not causing bottle necking of network.

\section{HSRP Disadvantages}

HSRP has a slow recovery time speed. The recovery time speed takes up to three second. For this kind of protocol three second is intolerable for real time traffic. For example, voice over IP traffic. HSRP

HSRP also has a low security. HSRP has two main issues with its security related problem. The first being it case by the router's incorrect configuration. This can make several routers be active or none of it being active. The second security problem it has is related to hostile user. If a hostile user is willing to cause Denial of Service or to seize confidential traffic. If an untrusted or unknown user is able to configure a device to take control the HSRP active router, it is a major security threat. However, with the combined use of Time To Live (TTL) along with local multicast address 224.0.02 it makes it extremely tough to actually attack HSRP if not being connected directly to the network.

\section{GLBP (Gateway Load Balancing Protocol) GLBP Advantages}

The first advantage of GLBP is that it efficiently uses its network resources. Several paths upstream can be utilized at the same time from the gateway.

It also has high availability. GLBP offers improved redundancy removing single point of failure of the first- hop gateway. GLBP can use the enhanced object tracking feature to ensure redundancy implementation mirrors network capabilities. This feature can also be used for HSRP and VRRP [8].

GLBP also has an automatic load balancing feature. The offnet traffic is shared among gateways that are available on a per-host basis, following the load- balancing algorithm that is defined.

GLBP also has a lower administration costs. This is because all the host on the subnet uses the same default gateway while load balancing is still able be accomplish. Making several gateway and groups not needed.

\section{GLBP Disadvantages}

Another disadvantage of GLBP is that it is cisco proprietary protocol. Meaning that is only compatible with Cisco products, this would mean that you would have to purchase Cisco products if you want to use this protocol.

GLBP is also a very complex protocol. Its complexity of network management is higher because of the high number of configurable parameters that are taken into consideration.

\section{METHODOLOGY}

Methodology that use in this research is interview. The advantage of doing interview is can get more extra details on the spot while doing interview. Furthermore, answer that get from interview is more accurate. Interview as methodology in this research is because it is difficult to get people who know FHRP. Normally who know this only people who work in network field. Therefore, in this research will interview 3 network experts and from there to gather data.

The data is to be collected via the following methodology as summarized in Table 4 below. 
Table 4 : Research Methodology [3][6]

\begin{tabular}{|c|c|}
\hline Research Dimension & $\begin{array}{c}\text { Explanatory Sequential } \\
\text { Design }\end{array}$ \\
\hline Research Methodology & Qualitative Reasoning \\
\hline Research Methods & $\begin{array}{c}\text { Personalized Interview } \\
\text { Simulation Testing }\end{array}$ \\
\hline
\end{tabular}

\section{Simulation Testing}

Network simulation is use a software that create a virtual environment that contain most of the equipment and tools to do network testing and design. So without using real machine able to encounter and predict some error before using real machine.

\section{RESULTS AND FINDINGS}

According out research we found that among all the FHRP GLBP is the best solution for medium size enterprise. GLBP is the best solution for every Medium Size company. It is because, there are some company like company that do animation normally the file they use are up to few TB per project. Therefore, Load Balancing is required. On top of these, among HSRP, VRRP and GLBP, GLBP is the only first hop redundancy protocol that able to do redundancy and load balancing [12]-[16]. GLBP is also the one most cost efficient because while doing redundancy all the router also running compare with VRRP and HSRP while 1 router running another router is only waiting GLBP is utilized the resources [17][20].

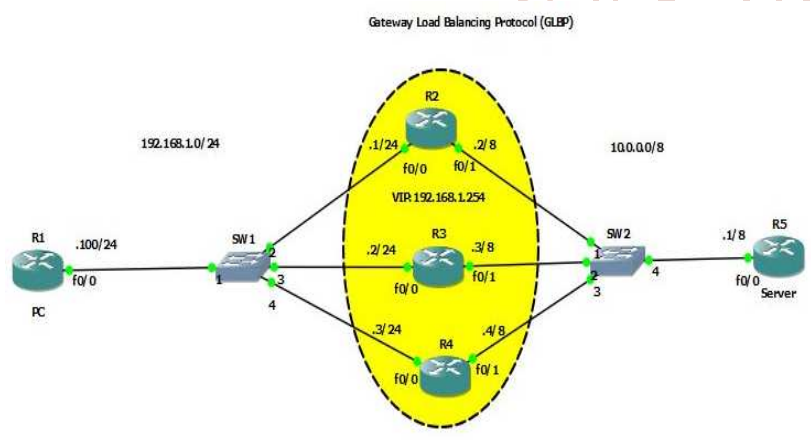

Figure 1: Network Topology

Figure above is GLBP network topology design by using GNS3, a network simulation tool.

Amount of network expert who thinks redundancy and load balancing is not required.

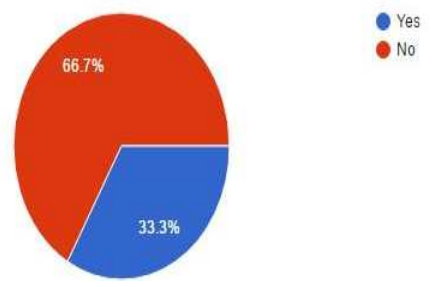

Figure 2: Certification Selection Preference

Based on network expert's opinion, $33.3 \%$ says that it is not necessary to have redundancy and load balancing function. However, $66.7 \%$ says that it is necessary for a medium sized enterprise. The reason for why the expert say it is not necessary is because it uses a layer 3 switch and also have a very low rate of failure.
Amount of network expert who thinks cost is the most important factor in choosing protocol for a medium sized enterprise.

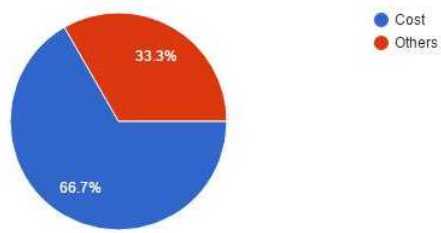

Figure 3: Certification Selection Preference

The figure above states that one of the most important factor in determining the protocol to choose is to think of the cost of the protocol. This is based on the survey done, $66.7 \%$ expert thinks that cost is the main factor. On the other hand, there is a $33.3 \%$ that does not agree with that statement. The reason as to why the expert say that cost is not the main factor is because he says it does not cost much.

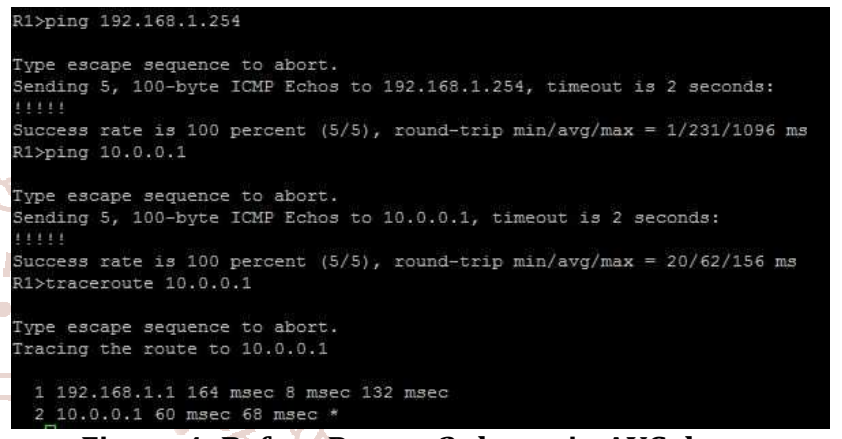

Figure 4: Before Router 2 the main AVG down

In this experiment there are 5 routers in use. Router $1=\mathrm{R} 1$. $\mathrm{RI}$ is the host and acts like a PC whereas Router 5 acts like a server. From router 2 to router 4 these router does redundancy and load balancing. From the figure above we can know that before router two is down, R1 ping to virtual IP and R5 is working fine and from traceroute, we are able to see that R1 is using R2's path to reach R5.

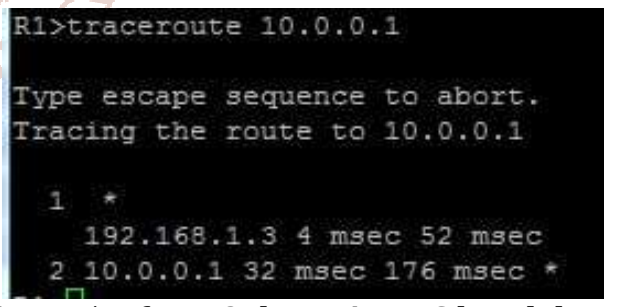

Figure 5: After R2 the main AVG breakdown

In figure 5, it shows that after R2 breakdowns by shutting down the interface that is connected with R1. This is able to seen from the picture trace route R1 using R4's path to reach R5.

\section{CONCLUSION AND FUTURE WORKS.}

In conclusion, technology is getting more advance nowadays and network plays a very important role in connection and communication. Therefore, to overcome network availability issue for medium size enterprise, GLBP is a very good redundancy protocol for Medium Size Enterprise.

"Energizing ICT infrastructure for Malaysia competitiveness during economic turbulence" Error! Reference source not found. become a crucial and avoidable consideration for the MSE. 
Malaysia is developing and improving daily, we hope that in the near future there will be more Medium Size Enterprise to apply GLBP protocol in their company to achieve higher availability network.

\section{REFERENCE}

[1] Gunsburg, Y. (2013). What are the differences between VRRP and HSRP? Retrieved July 12, 2016, from http://networkengineering.stackexchange.com/questio ns /792/what-are-the-differences-between-vrrp-andhsrp

[2] Kang, C. M., JosephNg, P.S. \& Issa, K. (2015), A study on integrating penetration testing into the information security framework for Malaysian higher education institution, International Conference on Mathematical Sciences and Computing Research (ISMSC), pp. 156-161

[3] JosephNg, P.S. (2018), EaaS Optimization: Available yet hidden information technology infrastructure inside medium size enterprise, Technology Forecasting and Social Changes, 132(7), pp. 165-173

[4] JosephNg, P.S. et al., (2016), EaaS : Available yet Hidden Infrastructure inside MSE, ACM International Conference on Network, Communication, and Computing, Kyoto, Japan, 2016, vol. 17, no. 21, pp. 17-21.

[5] JosephNg, P. S. \& Kang, C. M. (2016), Beyond Barebone Cloud Infrastructure Services: Stumbling Competitiveness During Economic Turbulences, Journal of Science \& Technology, 24(1), pp. 101-121.

[6] JN, P.S. et al., (2016), Exostructure Services for Infrastructure Resources Optimization, Journal of Telecommunication, Electronic and Computer Engineering, 8(4), pp. 65-69

[7] Joseph, N.P.S.; Choo, P.Y.; Wong, S.W.; Phan, K.Y. \& Lim, E.H. (2012), Hibernating ICT infrastructure during rainy days, Journal of Emerging Trends in Computing \& Information Sciences, 3(1); pp. 112-116

[8] Dubey, P., Sharma, S., \& Sachdev, A. (2013). Review of First Hop Redundancy Protocol and Their Functionalities. International Journal of Engineering Trends and Technology (IJETT).Soon, J.N.P. et al., (2013), Implementing of Virtual Router Redundancy Protocol in a Private University, Journal of Industrial and Intelligent Information, 1(4), pg 255-259

[9] S. K. Batumalai et al., (2015), IP Redundancy and Load Balancing With Gateway Load Balancing Protocol, Int. J. Sci. Eng. Technol., 4(3), pp. 218-222
[10] A. M. A. Mohamed et al., (2015), Hot Standby Router Protocol for a Private University in Malaysia, Int. J. Sci. Eng. Technol., 4(3), pp. 172-174

[11] S. H. R. Abdullaa et al., (2013), Implementing of Virtual Router Redundancy Protocol in a Private University, Journal of Industrial and Intelligent Information, 1(4), pp. 255-259

[12] Wilson, J. (2013). Comparing HSRP versus VRRP - same thing only different? Retrieved July 12, 2016, from Router Freak, http://www.routerfreak.com/comparinghsrp-versus-vrrp-same-thing-only-different

[13] Siew, J. X. et al., (2016). ECO QR Car Park System. International Journal of Scientific Engineering and Technology, 6(5), 176-180.

[14] L. Lim et al., (2016) "ScareDuino: Smart Farming with IOT," International Journal of Scientific Engineering and Technology, 6(5), 207-210.

[15] EdwinCheong, L. T. et al., (2017), JomNetwork: Reaffirming Resource Allocation through Network Monitoring, Journal of Information Systems Research and Innovation, 11(2), pp. 16-22

[16] Chris Foo, X.J., et al. (2017), JomNetwork: Medium Size Entreprise, Don't They Also Need Network Redundancy, Journal of Information Systems Research and Innovation, 11(2), pp. 1-5

[17] fretwell, J. (2012, May 9). HSRP vs VRRP vs GLBP 42814 - the Cisco learning network. Retrieved July 12, 2016, from Cisco Learning Network, https://learningnetwork.cisco.com/thread/42814

[18] Onart, A., \& Srikanth, A. (2003, January 31). VRRP Overview. Retrieved July 12, 2016, from inform it, http://www.informit.com/articles/article.aspx?p=3071 2

[19] Raj, A. (2011, June 2). Virtual Router redundancy protocol. Retrieved July 12, 2016, from Arvind S Raj's Blog, https://arvindsraj.wordpress.com/2011/06/02/virtual -router-redundancy-protocol/

[20] N, A. (2011). First Hop Redundancy protocol comparison (HSRP, VRRP, GLBP) with the diagram. Retrieved July 12, 2016, from Cisco networking center, http://cisconetworkingcenter.blogspot.my/2013/01/fir st-hop-redundancy-protocol.htm 